\title{
Electron Density Profiles in Cylindrical Plasmas From Microwave Refraction Data
}

\author{
B. A. Aničin ${ }^{1}$ \\ Department of Electronic and Electrical Engineering, University of Sheffield, England
}

(Received September 24, 1964; revised December 17, 1964)

\begin{abstract}
Electron density profiles in the afterglow of a stabilized linear pinch have been measured using the refractive properties of the plasma column. The deflection angle of a microwave beam refracted by the discharge is observed as a function of beam position. The resulting data lead to an integral equation for the electron density distribution, which is reducible to the Abel integral equation. The electron density is derived from the experimental data by inverting this last equation.

The method has been checked using direct transmission and interferometric measurements.
\end{abstract}

\section{Introduction}

Various branches of plasma physics, as well as the diverse applications of this science, call for a detailed knowledge of the electron density distributions occurring in a number of experimental situations.

Electromagnetic waves in a plasma are affected by the electrons present in it, so that microwave and optical diagnostic techniqes lead to data about electron densities. Present day sources in this part of the electromagnetic spectrum are characterized by a relatively narrow band, which prohibits the use of well-known ionospheric techniques employing large frequency excursions in the $10^{12}-10^{16} \mathrm{el} / \mathrm{cm}^{3}$ density range [Budden, 1961].

The problem of electron density profile measurement at a fixed frequency has been attacked in the past both theoretically and experimentally. The method described by Kharadly [1963] was developed for a plane stratified medium, and consists in measuring the distance between the incident and emergent beams as a function of the angle between the incident beam and the normal to the strata (fig. la). The method can also be applied to a cylindrical discharge, the ray path in figure la being contained in a meridian plane. The method can be shown to lead to unique electron density profiles, provided the profile is a monotonic function of position.

The method proposed by Shmoys [1961] makes use of the refractive properties of the plasma, the deflection angle $\theta$ being recorded as a function of the offset distance $b$ between the incident ray and the midplane of the discharge. The electron density distribution can be deduced uniquely from the $\theta(b)$ dependence (fig. lb), again provided the profile is a monotonic function of position. A common characteristic of both methods is that the density profile is deduced from a set of measured geometrical quantities at a single microwave frequency. In both methods it is necessary to make assumptions about the nature

On leave of absence from the Boris Kidrich Institute of Nuclear Sciences, Belgrade. of the electron distribution. Working in the meridian plane, one must assume that the density does not change along the strata and this requires the use of a sufficiently long tube. Axial symmetry is assumed in the transverse plane method, and measures have to be taken to ascertain the correctness of this assumption.

A similar approach was adopted by Wort [1963] who limited himself to the case of a parabolic electron density and applied this theory to the diagnostics of a statistically distributed bundle of plasma columns in a turbulent plasma.

This paper deals with the application of the transverse plane method to the measurements of electron density profiles in the afterglow of a linear pinch discharge. Section 2 deals with the theory of the experiment i.e., the calculation of density profiles from refraction data. It is essentially the same theory as that by Keller et al. [1956], except for the fact that the number of integrations in the final result has been reduced from two to one. The experiment itself is described in section 3.
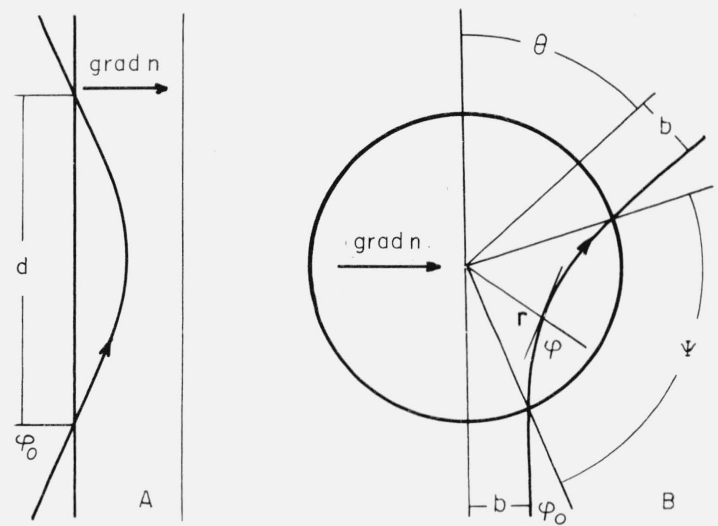

Figure 1. (a) Plane stratified medium.

(b) Cylindrically stratified medium. 


\section{Theory}

The ray trajectory is derived from Snell's law which has the form

$$
I(r) r \sin \varphi=\text { const. }
$$

in cylindrical coordinates; $I(r)$ is the refractive index, assumed to be a function of $r$ only, $\varphi$ is the angle between the ray trajectory and the radial direction. Now

$$
\frac{1}{r} \frac{d r}{d \theta}=\operatorname{ctg} \varphi
$$

which, combined with (1), gives the differential equation of the trajectory. We assume a collisionless plasma, so that the index of refraction can be expressed through the electron density only

$$
I^{2}(r)=1-\frac{n}{n_{c}},
$$

where $n_{c}$ is the critical electron density corresponding to the frequency of the microwave signal

$$
n_{c}=m \epsilon_{0} \omega^{2} / e^{2} .
$$

Combining (1), (2), and (3) and integrating, we get the expression for the deflection angle $\theta(b)$ (fig. lb)

$$
\theta(\dot{b})=\pi-2 \varphi_{0}=2 \int_{x_{0}}^{1} \frac{d x}{x \sqrt{\left(\frac{a}{b}\right)^{2} x^{2}\left(1-\frac{n}{n_{c}}\right)-1}}
$$

which is derived by Shmoys [1961]. $a$ is the radius of of the plasma column, i.e., that radius at which the electron density drops to zero; $b$ is the offset distance defined in figure $1 b$; as $\varphi_{0}=\sin ^{-1} \frac{b}{a}$ is the initial angle between the ray and radius vector (at $r=a$ ); $x=\frac{r}{a}$ is the normalized radius, $x_{0}$ is the normalized radius at the point of closest approach; i.e., the smallest real root of

$$
\left(\frac{a}{b}\right)^{2} x^{2}\left(1-\frac{n(x)}{n_{c}}\right)-1=0 .
$$

Equation (4) is an integral equation involving the electron density distribution. In the case of the plane st atified medium, one is also led to an integral equation for the density profile which can be reduced to an equation of the Abel type. To increase the similarity between the two (mathematically different) cases, we introduce the variables

$$
\begin{gathered}
\Psi(b)=\pi-2 \varphi_{0}-\theta(b), \\
\sin \varphi_{0}=\frac{b}{a} .
\end{gathered}
$$

If the observed quantities are $a \Psi\left(\varphi_{0}\right)$ and $\varphi_{0}$, the two experiments become identical for large values of $a$.

Equations (6) and (7) enable us to bring (4) in the form

$$
\frac{\Psi(b)}{2 \sin \varphi_{0}}=\int_{x_{0}}^{1} \frac{d x}{x \sqrt{x^{2}\left(1-\frac{n}{n_{c}}\right)-1+\cos ^{2} \varphi_{0}}} .
$$

Introducing the auxiliary variables

$$
\begin{aligned}
& Y=\frac{\Psi(b)}{2 \sin \varphi_{0}}, \\
& U=\cos ^{2} \varphi_{0}, \\
& V=1-x^{2}\left(1-\frac{n}{n_{c}}\right)
\end{aligned}
$$

we bring (4) to the standard Abel form

$$
Y(U)=-\int_{0}^{U} \frac{\frac{1}{x} \frac{d x}{d V}}{\sqrt{U-V}} d V .
$$

The lower limit in the last equation is zero as

$$
V(1)=1-1^{2}\left(1-\frac{n(1)}{n_{c}}\right)=0
$$

since we assume $n(1)=0$. If $n(1)$ is finite, (4) must be modified (invoking Snell's law) to take into account the finite refraction at the plasma-free space boundary.

Equation (12) is of the Abel type and has the inversion [Hamel, 1949]

$$
\frac{1}{x} \frac{d x}{d W}=-\frac{1}{\pi} \frac{d}{d W} \int_{0}^{W} \frac{Y(U)}{\sqrt{W-U}} d U
$$

$W$ is a parameter to be substituted by $V$ after integration and differentiation. The last equation can be integrated between the limits 0 and $W$ :

$\log x(W)-\log x(V=0)=-\frac{1}{\pi} \int_{0}^{W} \frac{Y(\boldsymbol{U})}{\sqrt{W-U}} d U$.

In a penetrable plasma $\left(\frac{n}{n_{c}}<1\right), \quad V=1-x^{2}\left(1-\frac{n}{n_{c}}\right)$ and $x^{2}\left(1-\frac{n}{n_{c}}\right)$ can be 1 only if $x=1$ and $n=0$. In an impenetrable plasma the same applies to the subcritical region where $\frac{n}{n_{c}}<1$. For the overcritical region $1-\frac{n}{n_{c}}<0$ and $V$ is always positive. So the second term-log $x(V=0)$ at the left-hand side of (14) is always zero, which gives

$$
x(W)=\exp \left\{-\frac{1}{\pi} \int_{0}^{W} \frac{Y(U)}{\sqrt{W-U}} d U\right\} .
$$


Note that only one integration has to be performed in (15). It gives the answer to the problem of finding the density distribution from refraction data given in the form of a $Y(U)$ function; $x(V)$ can be computed from (15) taking arbitrary values of $V$ and computing the corresponding values of $\frac{n}{n_{c}}$ from (11).

Let us check (15) in the simplest case of no electron density. It is evident that $\theta(b)=0$ and consequently $\Psi=\pi-2 \varphi_{0}$ which gives

$$
\begin{aligned}
Y(U)=\frac{\Psi}{2 \sin \varphi_{0}}=\frac{\pi-2 \varphi_{0}}{2 \sqrt{1-\cos ^{2} \varphi_{0}}}=\frac{\pi-2 \operatorname{arc} \cos \sqrt{U}}{2 \sqrt{1-U}} \\
Y(U)=\frac{\arcsin \sqrt{U}}{\sqrt{1-U}} .
\end{aligned}
$$

The $x(W)$ dependence is obtained from (15):

$x(W)=\exp \left\{-\frac{1}{\pi} \int_{0}^{W} \frac{\arcsin \sqrt{U}}{\sqrt{(1-U)(W-U)}} d U\right\}$

Now, it can be proved that

$$
\int_{0}^{W} \frac{\arcsin \sqrt{U}}{\sqrt{(1-U)(W-U)}} d U=-\frac{\pi}{2} \log (1-W)
$$

giving, after substitution of $W$ by $V$

$$
x(V)=\sqrt{1-V} .
$$

The electron density is found from (11):

$$
\begin{gathered}
V=1-(1-V)\left(1-\frac{n}{n_{c}}\right) \\
\text { i.e., } n=0 .
\end{gathered}
$$

The general equation (15) can be simplified using the integral (18). From (6) and (15) we get

$$
\begin{aligned}
x(W)=\exp \{- & \frac{1}{\pi} \int_{0}^{W} \frac{\pi-2 \varphi_{0}}{\sqrt{(1-U)(W-U)}} d U \\
& \left.+\frac{1}{2 \pi} \int_{0}^{W} \frac{\theta(b)}{\sqrt{(1-U)(W-U)}} d U\right\} .
\end{aligned}
$$

The first integral is the result (18), and we get

$$
x(W)=\sqrt{1-W} \exp \left\{\frac{1}{2 \pi} \int_{0}^{W} \frac{\theta(b)}{\sqrt{(1-U)(W-U)}} d U\right\} .
$$

As $\theta$ is measured as a function of $b$ we obtain another useful expression by introducing $s=\sin \varphi_{0}=\frac{b}{a}$ instead of $U=\cos ^{2} \varphi_{0}$ as the variable. To simplify the result we put $s=\sqrt{1-U}, w=\sqrt{1-W}$ and get

$$
\begin{gathered}
x(w)=w \exp \left\{\frac{1}{\pi} \int_{w}^{1} \frac{\theta(s)}{\sqrt{s^{2}-w^{2}}} d s\right\}, \\
\frac{n}{n_{c}}=\frac{x^{2}-v^{2}}{x^{2}} .
\end{gathered}
$$

It should be observed that the integration in (2l) is elementary for a polynominal $\theta(s)$. The actual procedure of interpreting the experimental data involved a polynomial of second degree.

\section{Experiments}

The basic idea of the experiment is shown in figure lb. Figure 2 is a schematic diagram of the microwave setup. A narrow microwave beam from the transmitting antenna is refracted by the plasma column and picked up by the receiving antenna. The transmitting horn is mounted on a microwave bench allowing lateral displacement of the incident beam. The receiving horn slides on another microwave bench and is always displaced for the same distance $b$ as the transmitting horn. This second bench is fastened to a turntable fitted with an angular dial permitting the reading of the angle $\theta$.

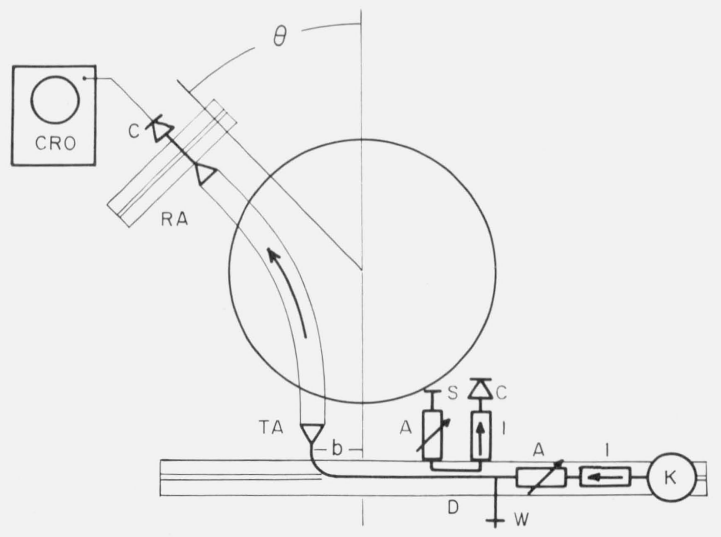

Figure 2. Microwave setup.

$$
\begin{aligned}
& \text { A - attenuator. } \\
& \text { I - ferrite isolator. } \\
& \text { K - klystron. } \\
& \text { D - directional coupler. } \\
& \text { W - wavemeter. } \\
& \text { C - crystal. } \\
& \text { S - short circuit. } \\
& \text { TA - transmitting antenna. } \\
& \text { RA - receiving antenna. } \\
& \text { CRO - cathode ray oscilloscope. }
\end{aligned}
$$




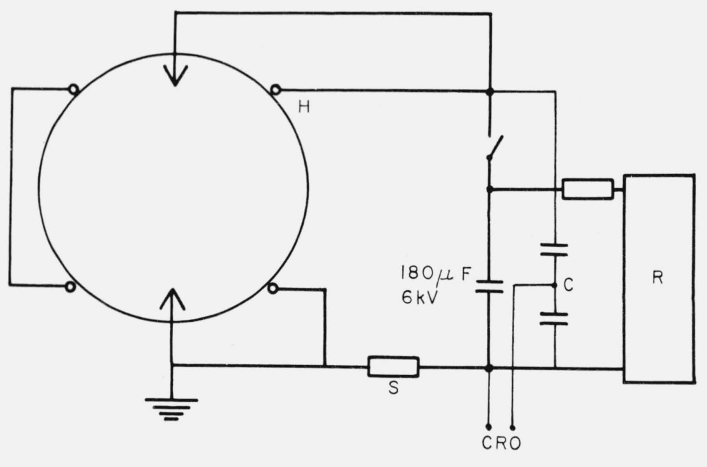

FigURE 3. Linear pinch equipment.

$\mathrm{R}$ - rectifier unit.
$\mathrm{H}$ - Helmholtz coils.
$\mathrm{S}$ - shunt.
$\mathrm{C}$ - capacitance divider.

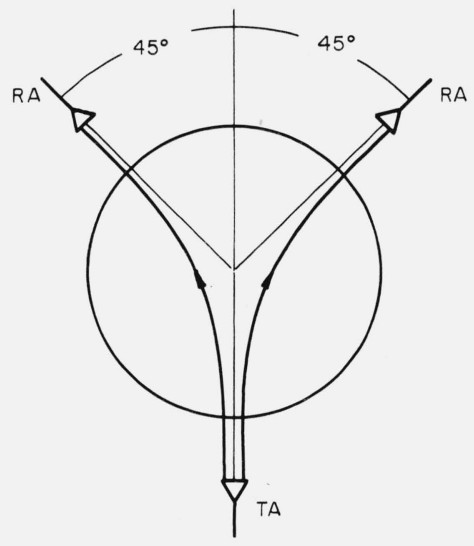

Figure 4. Setup for checking axial symmetry.

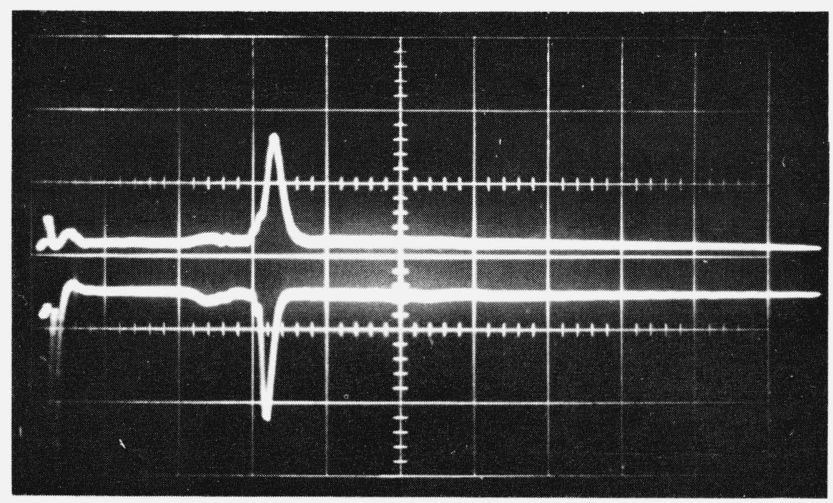

Figure 5. Check of axial symmetry.

The signal of the right-hand side horn is negative. The time scale is 0.5 $\mathrm{msec} / \mathrm{cm}$, the voltage scale is $0.05 \mathrm{~V} / \mathrm{cm}$.
The plasma was produced by discharging a condenser bank (180 $\mu \mathrm{F}, 6 \mathrm{kV}$ max.) through argon at 75 mtorr pressure, and hydrogen at 107 mtorr. A spherical vessel $70 \mathrm{~cm}$ in diameter fitted with pointed electrodes was used. The electrodes were fed through a large inductive loop resulting in a damped oscillatory current waveform, with a period of approximately $200 \mu \mathrm{sec}$ and peak currents of 10,000 A. An A.E.I. ignitron, type BK24A, was used as the switching element.

It was found essential to use a stabilizing axial magnetic field during the pinch process. This field (fig. 3) was produced by passing $10 \mathrm{kA}$ currents through a pair of single-turn Helmholtz coils, and had a maximum value of $200 \mathrm{G}$. The formative time lag of the main discharge is of the order of $20 \mu \mathrm{sec}$ during which period the axial magnetic field is allowed to increase, being trapped at the start of the main discharge. Without this precaution the plasma in the afterglow was found to be nonreproducible and axially nonsymmetric. As axial magnetic fields are known to suppress instabilities in linear pinches, the magnetic field is considered to stabilize the initial conditions of the discharge, leading to an axially symmetric plasma in the afterglow.

As the main assumption in the theory of the experiment is the axial symmetry of the plasma column, this was checked first. The arrangement is shown in figure 4. Two receiving horns at $45^{\circ}$ from the direction of the transmitting horn pick up the refracted microwave signals. It was found that both left and right signals occurred at approximately the same time (fig. 5), which ascertains axial symmetry at that particular instant of time.

Another essential assumption is the reproducibility of the discharge. Values of $\theta$ corresponding to different values of the offset distance $b$ are measured in different firings, so that consistent results can be expected only if the electron density is sufficiently reproducible from one discharge to another. Reproducibility was constantly checked by observing the pulse shape and the peak of pulse time in the symmetry experiment. Even with an axial magnetic field the first few discharges were dissimilar to all other subsequent firings. Discharges took place at regular time intervals (1 min). After at most 10 discharges, the pulses did not change in shape and time. If the apparatus was not operated for $15 \mathrm{~min}$, this effect was not observed, but an interval of $1 \mathrm{hr}$ was sufficient to cause a few initial pulses to be irregular. This effect was ascribed to the gases occluded in the electrodes.

All experiments have been carried out at the fixed wavelength of $8.54 \mathrm{~mm}$. Both transmitting and receiving aerials were phase-corrected horn-lens combinations, designed on the basis of geometrical optics. The lens diameter was $70 \mathrm{~mm}$. A lens diameter of the order of $10 \lambda$ is essential, as smaller lenses yield unreasonably diverging beams.

Measurements at a prescribed time in the afterglow can be performed by observing the angle $\theta$ as a function of time with the offset distance $b$ as a variable 
parameter and then determining $\theta$ as a function of $b$ at a given time from the resulting curves. A different procedure was adopted here however. Both receiving and transmitting horns are first set to a given offset distance $b$. Then the turntable is manipulated in the course of firing until the peak of the received microwave pulse falls at the required instant of time on a cathode ray oscilloscope. The sensitivity of the method depends upon the narrowness of the received pulses. The pulse shape changes appreciably with time in the afterglow and also with the bank voltage. Best results in argon have been obtained at $1600 \mu$ sec after the start of the discharge with a bank voltage of $3.5 \mathrm{kV}$. Direct transmission experiments indicate that the discharge was critical at $1500 \mu \mathrm{sec}$.

The measured $\theta(b)$ curve is the solid line in figure 6 . Note that there is no refraction for $b / a>0.35$. The curve is the mean of 5 measurements, the maximum deviation in the results being $3^{\circ}$. To obtain the electron density profile a parabola of the type

$$
\begin{aligned}
& \theta=\theta_{0}\left[1-\left(\frac{s}{s_{0}}\right)^{2}\right], s<s_{0}, s=\frac{b}{a}, \\
& \theta=0, s>s_{0}
\end{aligned}
$$

was fitted to the experimental curve by least square methods, giving

$$
\theta_{0}=44^{\circ}, \quad s_{0}=0.347
$$

The dotted line in figure 6 is the parabolic approximation. Equations (21) and (22) have been used to compute the electron density profile, giving

$$
\begin{aligned}
x(v)= & v \exp \left\{\frac { \theta _ { 0 } } { \pi } \left[\left(1-\frac{v^{2}}{2 s_{0}^{2}}\right) \log \frac{s_{0}+\sqrt{s_{0}^{2}-v^{2}}}{v}\right.\right. \\
& \left.\left.-\frac{\sqrt{s_{0}^{2}-v^{2}}}{2 s_{0}}\right]\right\}, \frac{n}{n_{c}}=\frac{x^{2}-v^{2}}{x^{2}} .
\end{aligned}
$$

The profile obtained from the last two equations is the solid line in figure 7 . The fact that $n=n_{c}$ at $r=0$ is a result of the failure of geometrical optics near the axis of the plasma column. In ray theory any $\theta(b)$ curve with a finite value of $\theta(0)$ leads to $n=n_{c}$ at the axis.

The anomaly may be understood by referring to figure 5 in Shmoys [1961]. This is a $\theta(b)$ curve for a parabolic distribution function, and it is fairly reasonable to assume that the electron density near the center of the plasma column is parabolic. We see from the curve that most of the electromagnetic energy in the central part of a finite beam would be scattered between $\theta=0$ and $\theta=\theta_{\max }$, the outer portion of the beam forming a caustics at $\theta=\theta_{\max }$. The maximum energy flow is not to be expected near $\theta=0$, although the plasma is penetrable.

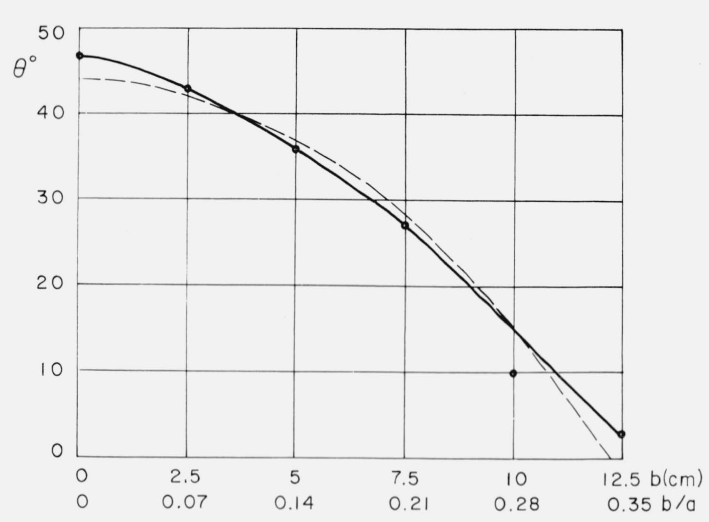

Figure 6. Measured $\theta(\mathrm{b})$ dependence.

Argon pressure 75 mtorr, bank voltage $3.5 \mathrm{kV}$. The curve is the mean of 5 measurements the maximum deviation in the results being $3^{\circ}$. Note that there is no refraction for $b / a>0.35$. The dotted line is a parabolic approximation of the experimental data obtained by least square methods, $\theta=\theta_{0}\left[1-\left(s / s_{0}\right)^{2}\right], \theta_{0}=44^{\circ}, s_{0}=0.347, s=\frac{b}{a}$

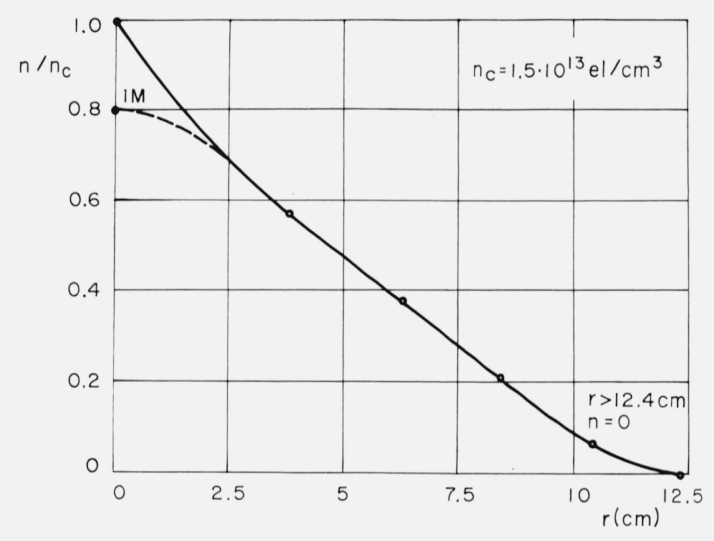

FIGURE 7. Electron density profile in the afterglow of a linear pinch in argon.

The solid line is obtained by inverting the integral equation for the electron density distribution, assuming a parabolic $\theta(b)$ dependence (fig. 6). The point labeled IM is a result of interferometric measurements. The dotted line is a parabola, representing the density near the column center.

The electron density at $x=0$ was derived from interferometric measurements. The interferometer shown in figure 2 is set to measure the phase shift of the microwave signal reflected from a plane mirror at $b=0$. Figure 8 is a logarithmic plot of phase shift versus time. We deduce from this figure that the electron density at the axis was $0.8 n_{c} 100 \mu \mathrm{sec}$ after the plasma was critical. This is the point labeled IM in figure 7. The broken line in the same figure is a parabolic continuation starting at that point.

For $b / a>0.35$ the plasma is not sufficiently refractive to cause any measurable deflection of the beam. To verify this result an independent check of the experiment was provided. This is essentially a direct transmission test. The transmitting and receiving horns are located opposite each other on a line $b=$ const., and the time during which the microwave signal is cut off is recorded. 


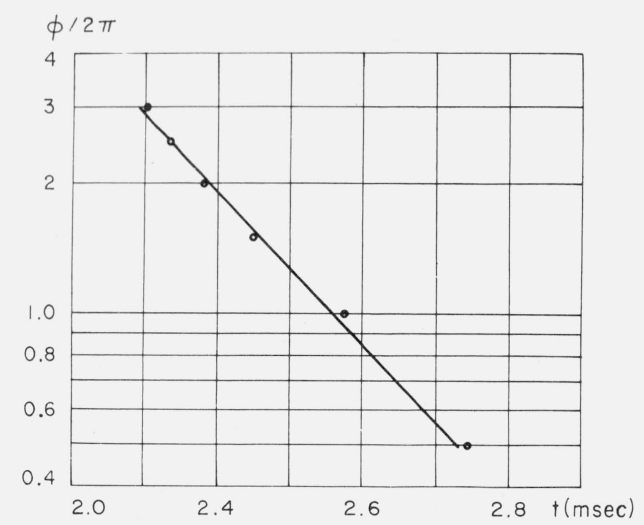

FIG (JRE 8. Phase shift versus time from interferometric measurements The average electron density, proportional to phase shift, drops for 20 per cent in a $100 \mu \mathrm{sec}$ interval.

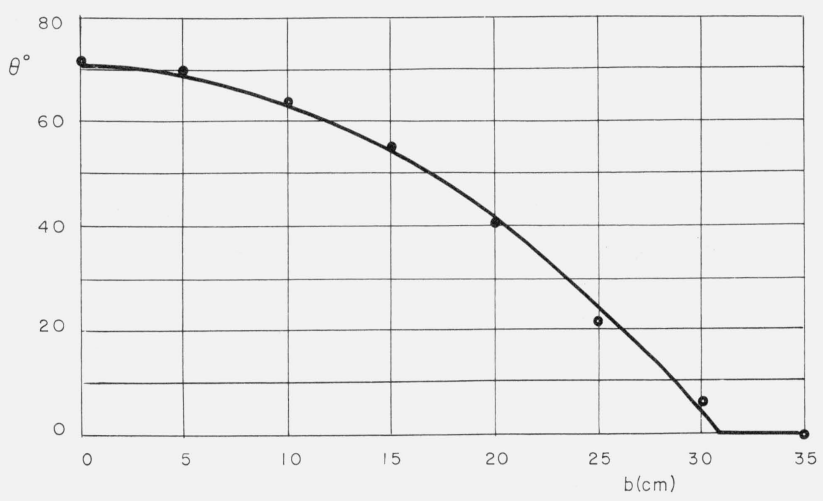

FiguRE 9. $\theta$ (b) measured in hydrogen.

Pressure 107 mtorr, bank voltage $4 \mathrm{kV}$. The points are experimental, the curve is the parabolic approximation.

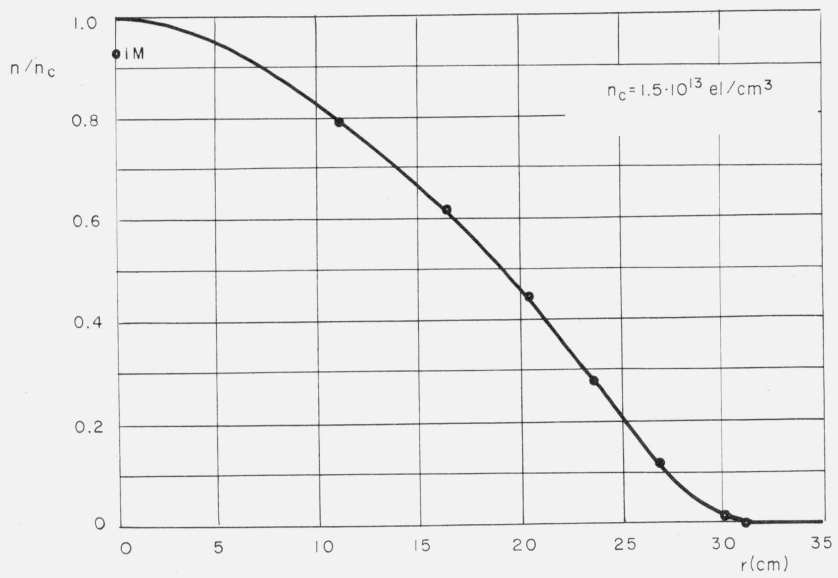

FiguRE 10. Electron density profile in the hydrogen discharge. The point labeled IM is the result of interferometric measurements.

This was done at $b=5,10,15,20,25$, and $30 \mathrm{~cm}$. The plasma does not perturb the microwave beam at 25 and $30 \mathrm{~cm}$. At 15 and $20 \mathrm{~cm}$ the plasma was found penetrable for periods sufficiently long to estimate the electron density as $<\frac{1}{100} n_{c}$ at $t=1600 \mu$ sec from the decay rate obtained from interferometric measurements. The fact that the microwave beam at the edge of the vessel is unperturbed during the discharge and the whole of the afterglow provides an experimental test of the assumption $n(1)=0$.

A similar experiment was carried out in hydrogen at 107 mtorr. Unlike argon, where the visible discharge was confined to a narrow column, the light from the hydrogen discharge came from a central area occupying $1 / 3$ of the vessel. Without the axial magnetic field the luminous discharge fills practically the whole of the vessel, but the reproducibility of the microwave cutoff time was not sufficient to permit measurements of profiles. With the axial magnetic field, best reproducibility and symmetry have been obtained at $4 \mathrm{kV}$ condenser bank voltage. The plasma is critical at $245 \mu$ sec. The $\theta(b)$ was measured at $250 \mu \mathrm{sec}$ after the application of the voltage pulse to the gap. The curve is shown in figure 9. The points are the mean of three measurements with a maximum deviation of $5^{\circ}$. The solid line is the parabolic approximation given by the equation $\theta=71-90 \mathrm{~s}^{2}$. The profile obtained from this approximation using (23) and (24) is given in figure 10 . For $r=0, n=n_{c}$ again, but there is no cusp at this point. It is a property of the parabolic approximation used that it leads to spiky profiles for $\theta(0)<60^{\circ}$. Irrespective of the character of the derivative of the profile curve near the axis the value of the electron density obtained in this region are not correct unless the beam width is smaller than the radius corresponding to the maximum in the theoretical $\theta(b)$ curve. According to interferometric measurements the electron density at the axis is $0.94 n_{c}$. This is the point labeled IM in figure 10 . The direct transmission test applied to the hydrogen discharge shows that the electron density at the wall is zero.

A suitable quantitative criterion for the applicability of ray optics is necessary both for the argon and hydrogen discharges. Experimentally one measures the maxima of the received pulses. In ray theory, these coincide with the central ray of the beam. Wave optics gives different positions for the maxima. The quantitative corrections to ray theory necessary from the standpoint of wave theory have been computed by A. L. Cullen [1965] for a plane stratified medium. It follows from his theory that the applicability of ray optics to the oblique incidence experiment in critical plasmas of the sizes encountered here is justified.

\section{Conclusions}

A microwave diagnostic method giving electron density profiles was applied to the afterglow plasma of a stabilized linear pinch. The deflection of a $8 \mathrm{~mm}$ microwave beam is measured as a function of beam position. A simplified theory of the experiment is presented. 
The ray theory version of the method is applicable only to very large, spatially slowly varying plasmas. With a finite microwave beam width difficulties occur near the center of the column where the deflection of a ray varies very much with ray offset distance. In a time varying plasma the method yields electron density profiles only in a short interval of time in which the plasma is sufficiently refractive, in the vicinity of the instant at which the center of the column is critical. The sensitivity of the method is affected by the shape of the received microwave pulses and the reproducibility of the discharge. The procedure does not require a great deal of axial space. On the other hand, the plasma column must possess symmetry of revolution, and this assumption must be checked.

The author acknowledges with appreciation valuable discussions of this problem with Professor A. L. Cullen and Dr. P. N. Robson of The Department of Electronic and Electrical Engineering of The University of Sheffield. Appreciation is expressed for the award of a grant by the Boris Kidrich Institute of Nuclear Sciences, Belgrade.

\section{References}

Budden, K. G. (1961), Radio waves in the ionosphere (Cambridge University Press, Cambridge, Eng.).

Cullen, A. L. (1965), On the point of emergence of a microwave beam entering a linearly graded plasma, Radio Sci. J. Res. 69D, No. 2, $177-178$

Hamel, G. (1949), Integralgleichungen. Einführung in die Lehre und Gebrauch, Springer Verlag, Berlin, p. 136.

Keller, J. B., I. Kay, and J. Shmoys (Apr. 1956), Determination of the potential from scattering data, Phys. Rev. 102, No. 2, 557.

Kharadly, M. M. Z. (July 1963), A new millimetre-wave method for determination of electron density profiles in a linear discharge, Proc. IEE 110, No. 7, 202.

Shmoys, J. (April 1961), 'Proposed diagnostic method for cylindrical plasmas, J. Appl. Phys. 32, No. 4, 689-695.

Wort, D. J. H. (1963), Refraction of microwaves by a plasma cylinder, Culham Laboratory Report CLM-R27. 